\title{
WAVELET-TYPE FRAMES AND WAVELET-TYPE BASES
}

\author{
M. M. SHAMOOSHAKY
}

Received 13 January 2005 and in revised form 17 September 2005

The concepts of basis and frame are studied in the classical literature of functional analysis, Fourier analysis, and wavelet theory in a wide range. In this paper, we consider an operator-theoretic approach to discrete frame theory on a separable Hilbert space. For this purpose, we define a special type of frames and bases, called wavelet-type frames and wavelet-type bases, obtained by acting with a family of bounded linear operators on some vectors, and then investigate the elementary properties of these concepts.

\section{Introduction}

The idea of this paper comes from wavelets theory. In classical functional analysis, a basis of a separable Hilbert space is a countable subset of $H$ with certain properties. In the wavelet theory, a basis is made by a countable family of unitary operators and a single number (or finite number) of vectors in $H$. In fact, if $U$ is a unitary system on $H$ and $\Psi$ is the mother wavelet corresponding to it, then $U \Psi$ is an orthonormal basis for $H$. In other words, the bases which one studies in the wavelet theory are of this form.

Hence generally, a frame $\left\{x_{n}: n \in \mathbb{I}\right\}$ for $H$ is called a wavelet-type frame if there exist a family $\left\{f_{i}: i \in \mathbb{D}\right\}$ of bounded operators on $H$ and a vector $h \in H$ such that $x_{i}=f_{i} h$ for each $i \in \mathbb{J}$. The vector $h$ is called a framer vector.

Similarly, a normalized tight frame (Riesz basis, orthonormal basis) $\left\{x_{j}: j \in \mathbb{I}\right\}$ is called a wavelet-type normalized tight frame (wavelet-type Riesz basis, wavelet-type orthonormal basis) if there exist a family $\left\{f_{i}: i \in \mathbb{V}\right\}$ of bounded linear invertible operators on $H$ and a vector $h \in H$ such that $x_{i}=f_{i} h$ for each $i \in \mathcal{J}$.

Our idea in this paper is based on the wavelet-type frames, which we organize as follows.

In Section 2, definitions and some elementary properties of framers and wavelet-type frames, basers and wavelet-type bases are given. Then, we will explain the relations between, framers and normal framers, normal framers and orthonormal basers, framers and Riesz basers, and finally, framers and the canonical dual of it. These results will be needed in Section 3. 
Section 3 is devoted to the study of the structure of framers and basers, by defining the concepts of complement, strong complement, strongly disjoint complement, and similarity. We will show that the complement of each wavelet-type frame is of wavelet-type. The same result will be proven for the strong complement, the strongly disjoint complement, and similarity. Also, the problem of construction of a baser from a framer on a Hilbert space will be considered in this section. Indeed by using a complement framer, we can find a (Riesz or orthonormal) baser.

\section{Elementary properties of framers and basers}

Let $H$ be a separable Hilbert space, and let $B(H)$ denote the algebra of all bounded linear operators on $H$. Let $\mathbb{\rrbracket}$ denote a generic countable (or finite) index set such as $\mathbb{N}, \mathbb{Z}$, and so forth.

In the notations, we follow [8].

Definition 2.1. Let $H$ be a separable complex Hilbert space and $B(H)$ the algebra of all bounded linear operators on $H$. If $U=\left\{f_{j}: j \in \mathbb{I}\right\}$ is a countable subset of bounded operators on $H$, and $\lambda \in H$, then the following hold.

(i) $\left(\left\{f_{j}\right\}, \lambda\right)$ is a framer on $H$ if $U \lambda=\left\{f_{j} \lambda: f_{j} \in U\right\}$ is a frame with frame bounds $A$, $B$, for $H$. Also $A, B$ are called framer bounds.

(ii) $\left(\left\{f_{j}\right\}, \lambda\right)$ is a tight framer on $H$ if the $f_{j}$ 's are invertible, for each $j \in \mathbb{J}$, and $U \lambda=$ $\left\{f_{j} \lambda: f_{j} \in U\right\}$ is a tight frame for $H$.

(iii) $\left(\left\{f_{j}\right\}, \lambda\right)$ is a normal framer on $H$ if the $f_{j}$ 's are invertible, for each $j \in \mathbb{J}$, and $U \lambda=\left\{f_{j} \lambda: f_{j} \in U\right\}$ is a normalized tight frame for $H$.

(iv) Finally, $\left(\left\{f_{j}\right\}, \lambda\right)$ is called (an orthonormal-Riesz) baser on $H$ if the $f_{j}$ 's are invertible, for each $j \in \mathbb{I}$, and $U \lambda=\left\{f_{j} \lambda: f_{j} \in U\right\}$ is (an orthonormal-Riesz) basis for $H$.

\subsection{Examples.}

(a) Let $H=l^{2}(\mathbb{N} \cup\{0\})$ and $T: l^{2}(\mathbb{N} \cup\{0\}) \rightarrow l^{2}(\mathbb{N} \cup\{0\})$ is defined by $T\left(x_{1}, x_{2}, \ldots\right)=$ $\left(0, x_{1}, x_{2}, \ldots\right)$, then $T$ is a bounded invertible operator with $\|T\|=1$. Set $T_{n}=T^{n}$ with

$$
T^{n}\left(x_{1}, x_{2}, \ldots\right)=(\underbrace{0,0, \ldots, 0}_{n \text { times }}, x_{1}, x_{2}, \ldots), \quad T_{0}=T, \quad \lambda=(1,0,0, \ldots),
$$

then $\left(\left\{T_{n}: n \in \mathbb{N} \cup\{0\}\right\}, \lambda\right)$ is an orthonormal baser on $H$.

(b) Let $H=L^{2}[0,1]$, define $T(x(t))=\int_{0}^{t} x(q) d q$ and by induction, $T^{n}(x(t))=$ $(1 / n) T^{n-1}(x(t))$, if $x(t)=1$, then $T(x(t))=t, T^{2}(x(t))=(1 / 2) t^{2}, \ldots, T^{n}(x(t))=$ $(1 / n !) t^{n}$, and $\left\{1, t,(1 / 2) t^{2}, \ldots,(1 / n !) t^{n}, \ldots\right\}$ is a tight frame (see [8]), so if we define $T_{n}=T^{n}, n \in \mathbb{N}$, then $\left(\left\{T_{n}: n \in \mathbb{N}\right\}, x(t)\right)$ is a tight framer on $H$.

(c) Let $\Psi$ be the mother wavelet corresponding to unitary system $U$ for Hilbert space $H$, then $(U, \Psi)$ is an orthonormal baser on $H$.

Theorem 2.2. Let $\left\{f_{n}: n \in \mathbb{J}\right\}$ be a normal framer on $H$. Then there exist a Hilbert space $K$ and a normal baser $U=\left\{T_{n}: n \in \mathbb{J}\right\}$ of $K$ such that $\theta f_{n} \theta^{*}=P T_{n}$, or $\theta f_{n}(x)=P T_{n}(\theta(x))$, 
$n \in \mathbb{J}$, where $\theta: H \rightarrow \theta(H)$ is a unitary operator and $P: K \rightarrow \theta(H)$ is the orthogonal projection of $H$ onto $\theta(H)$.

Proof. Since $\left\{f_{n}: n \in \mathbb{I}\right\}$ is a normal framer, there exists $h \in H$ such that $\left\{f_{n} h: n \in\right.$ 】\} is a normalized tight frame for $H$. Set $K=l^{2}(\rrbracket)$ and define $\theta_{0}: H \rightarrow K$ by $\theta_{0}(x)=$ $\left(\left\langle x, f_{n} h\right\rangle\right)_{n \in \mathbb{I}}$ as the usual frame transform which is an isometry. Thus, $H$ can be embedded into $K$ by identifying $H$ with $\theta_{0}(H)$. Since $\theta: H \rightarrow \theta_{0}(H) \subset K$ with $\theta(x)=\theta_{0}(x)$ is onto, so it is unitary. Define $T_{1}: K \rightarrow K$ as identity and

$$
T_{2}\left(\left(x_{n}\right)_{n \in \mathbb{I}}\right)=\left(0, x_{1}, x_{2}, \ldots\right), \ldots, T_{m}\left(\left(x_{n}\right)_{n \in \mathbb{I}}\right)=(\underbrace{0,0, \ldots, 0}_{m-1 \text { times }}, x_{1}, x_{2}, \ldots),
$$

then $\left\{T_{n}\right\}_{n \in \rrbracket}$ with $e_{1}=(1,0,0, \ldots)$ is an orthonormal baser on $K$. Since $P$ is a projection, so $P=P^{*}$, and we have $\left\langle\theta f_{m}(x), P T_{n}(\theta(x))\right\rangle=\left\langle P \theta f_{m}(x), T_{n}(\theta(x))\right\rangle=\left\langle\theta f_{m}(x), T_{n}(\theta(x))\right\rangle=$ $\left\langle f_{m}(x), f_{n}(\theta(x))\right\rangle=\left\langle\theta\left(f_{m}(x)\right), \theta f_{n}(\theta(x))\right\rangle$, hence $\left\langle\theta f_{m}(x), P T_{n}(\theta(x))-\theta f_{n}(\theta(x))\right\rangle=0$. But by definition of framer, we have $\left\langle\theta(y), T_{n}\left(e_{1}\right)\right\rangle=\left\langle y, f_{n}(h)\right\rangle$. On the other hand, since $f_{m}(h)$ 's span $H$, thus vectors $\theta f_{m}(h)$ span $\theta(H)$, so $\left(P T_{n} e_{1}-\theta\left(f_{n} h\right)\right) \perp \theta(H)$, and hence $\left(P T_{n} \theta(x)-\theta\left(f_{n}(x)\right)\right) \perp \theta(H)$. However, the range of $P$ is $\theta(H)$, and therefore $P T_{n} \theta(x)-$ $\theta\left(f_{n}(x)\right)=0$ and $P T_{n} \theta(x)=\theta\left(f_{n}(x)\right)$.

The relation between normal framers and orthonormal basers will be shown in the following theorem.

Theorem 2.3. Let $H$ be a Hilbert space, then $\left(\left\{f_{n}: n \in \mathbb{J}\right\}, h_{1}\right)$ is a normal framer on $H$ if and only if there exist a Hilbert space $M$ and a normal framer $\left(\left\{g_{n}: n \in \mathbb{J}\right\}, h_{2}\right)$ on $M$ such that $\left(\left\{f_{n} \oplus g_{n}: n \in \mathbb{I}\right\}, h_{1} \oplus h_{2}\right)$ is an orthonormal baser on $H \oplus M$.

Proof. By Theorem 2.2, there exist a Hilbert space $K$ and an orthonormal baser $\left(\left\{u_{n}: n \in\right.\right.$ I\}, $\left.e_{1}\right)$ on $K$ such that $\theta_{0}\left(f_{n}(x)\right)=P u_{n}\left(\theta_{0}(x)\right)$, where $\theta_{0}: H \rightarrow K$ is the frame transform corresponding to normalized tight frame $\left\{f_{n} h_{1}: n \in \mathbb{J}\right\}$, which is an isometry. Now set $M=(I-P) K$ and $g_{n}=(I-P) u_{n}$, where $I$ is the identity operator on $K$, then the set $\left\{g_{n} e_{1}: n \in \mathbb{J}\right\}$ is a normalized tight frame for $M$, hence $\left(\left\{g_{n}: n \in \mathbb{J}\right\}, e_{1}\right)$ is a normal framer on $M$ and $\left(\left\{\theta_{0}\left(f_{n}\right) \oplus g_{n}: n \in \mathbb{J}\right\}, \theta_{0}\left(h_{1}\right) \oplus h_{2}\right)$ is an orthonormal baser on $\theta_{0}(H) \oplus M$. Since $\theta_{0}: H \rightarrow K$ is an isometry, if we define $\theta: H \rightarrow \theta_{0}(H)$ by $\theta(x)=\theta_{0}(x)$, then $\theta$ is unitary, so $\theta \oplus I$ and $\theta^{-1} \oplus I$ are unitaries. Since $\left(\left\{\theta_{0}\left(f_{n}\right) \oplus g_{n}: n \in \mathbb{J}\right\}, \theta_{0}\left(h_{1}\right) \oplus h_{2}\right)$ is an orthonormal baser on $\theta_{0}(H) \oplus M$, hence

$$
\begin{gathered}
\left(\left\{\left(\theta^{-1} \oplus I\right)\left(\theta_{0}\left(f_{n}\right) \oplus g_{n}\right): n \in \mathbb{J}\right\},\left(\theta^{-1} \oplus I\right)\left(\theta_{0}\left(h_{1}\right) \oplus h_{2}\right)\right) \\
=\left(\left\{f_{n} \oplus g_{n}: n \in \mathbb{J}\right\}, h_{1} \oplus h_{2}\right)
\end{gathered}
$$

is an orthonormal baser on $\left(\theta^{-1} \oplus I\right)\left(\theta_{0}(H) \oplus M\right)=H \oplus M$ as required.

The next theorem show that a Riesz baser is the image of an orthonormal baser under a bounded invertible operator.

Theorem 2.4. Let $\left(\left\{v_{n}: n \in \mathbb{J}\right\}, \lambda_{1}\right)$ be a Riesz baser on Hilbert space $H$, then there exist a bounded invertible operator $\theta$ and an orthonormal baser $\left(\left\{u_{n}: n \in \mathbb{J}\right\}, \lambda_{2}\right)$ such that $\theta v_{n}(x)=$ 
$u_{n}(\theta(x))$. Likewise, a framer is the image of a normal framer under a bounded invertible operator.

Proof. Let $\left(\left\{v_{n}: n \in \mathbb{I}\right\}, \lambda_{1}\right)$ be a Riesz baser on Hilbert space $H$, then by the proof of Theorem 2.2, we have $\theta_{0}(H)=K$ and $\theta_{0}^{-1}(K)=H$, hence if $\left(\left\{u_{n}: n \in \mathbb{I}\right\}, \lambda_{2}\right)$ is the normal baser as mentioned in Theorem 2.2, then $\theta_{0}^{-1}\left(u_{n}\left(\theta_{0}(x)\right)\right)=v_{n}(x)$, or $\theta_{0} v_{n}(x)=u_{n}\left(\theta_{0}(x)\right)$.

Let $\left(\left\{u_{n}: n \in \mathbb{J}\right\}, h\right)$ be a normal framer on $H$ and $T$ a bounded linear invertible operator. Define $f_{n}(x)=T u_{n}(x)$, then for $x_{0} \in H$, we have $\sum_{j}\left|\left\langle x_{0}, f_{j}(h)\right\rangle\right|^{2}=\sum_{j}\left|\left\langle x_{0}, T u_{j}(h)\right\rangle\right|^{2}=$ $\left.\sum_{j}\left\langle T^{*} x_{0}, u_{j}(h)\right\rangle\right|^{2}=\left\|T^{*} x_{0}\right\|^{2}$.

But

$$
\left\|T^{*} x_{0}\right\| \leq\left\|T^{*}\right\|\left\|x_{0}\right\|=\|T\|\left\|x_{0}\right\| .
$$

Since $T^{-1}$ exists, so $T^{*}$ is invertible and $T^{*^{-1}}=\left(T^{-1}\right)^{*}$, thus $\left\|T^{*^{-1}}\right\|=\left\|\left(T^{-1}\right)^{*}\right\|=$ $\left\|T^{-1}\right\|$, hence

$$
\left\|x_{0}\right\|=\left\|T^{*^{-1}} T^{*}\left(x_{0}\right)\right\| \leq\left\|T^{-1}\right\|\left\|T^{*}\left(x_{0}\right)\right\|
$$

Now (2.4) and (2.5) imply that $\left\|T^{-1}\right\|^{-1}\left\|x_{0}\right\| \leq\left\|T^{*}\left(x_{0}\right)\right\| \leq\|T\|\left\|x_{0}\right\|$. Therefore, $\left\|T^{-1}\right\|^{-2}\left\|x_{0}\right\|^{2} \leq \sum_{j}\left|\left\langle x_{0}, f_{j}(h)\right\rangle\right|^{2} \leq\|T\|^{2}\left\|x_{0}\right\|^{2}$, hence $\left\{f_{n}(T h): n \in \mathbb{\rrbracket}\right\}$ is a frame with frame bounds $A \geq\left\|T^{-1}\right\|^{-2}$ and $B \leq\|T\|^{2}$, and consequently $\left(\left\{f_{n}: n \in \mathbb{I}\right\}, h\right)$ is a framer.

In Theorem 2.3, we showed that each normal framer is the direct summand of a normal baser. We will prove the same result for Riesz basers as follows.

Theorem 2.5. Let $\left(\left\{f_{n}: n \in \mathbb{J}\right\}, h\right)$ be a framer on Hilbert space $H$. Then there exist a Hilbert space $M$ and a normal framer $\left(\left\{u_{n}: n \in \mathbb{J}\right\}, \lambda\right)$ on $M$ such that $\left(\left\{f_{n} \oplus u_{n}: n \in\right.\right.$ ป\}, $h \oplus \lambda)$ is a Riesz baser on $H \oplus M$.

Proof. Since by Theorem 2.4 each framer is the image of a normal framer under a bounded invertible operator, there exist a normal framer $\left(\left\{v_{n}: n \in \mathbb{I}\right\}, \lambda_{1}\right)$ on $H$ and invertible operator $T: H \rightarrow H$ such that $T v_{n}(x)=f_{n}(x)$ for all $n \in \mathbb{J}$. So by Theorem 2.3, there are a Hilbert space $M$ and a normal framer $\left(\left\{u_{n}: n \in \mathbb{I}\right\}, \lambda_{2}\right)$ on $M$ such that $\left(\left\{\theta\left(v_{n}\right) \oplus u_{n}: n \in \mathbb{J}\right\}, \theta\left(\lambda_{1}\right) \oplus \lambda_{2}\right)$ is an orthonormal baser on $\theta(H) \oplus M$. Since $T$ and $\theta^{-1}$ are invertible, so $T \theta^{-1}$ is invertible, $\left(T \theta^{-1} \oplus I\right)\left(\theta\left(\lambda_{1}\right) \oplus \lambda_{2}\right)=T \lambda_{1} \oplus \lambda_{2}$, and $\left(T \theta^{-1} \oplus\right.$ I) $\left(\theta\left(v_{n}\right) \oplus u_{n}\right)=T\left(v_{n}\right) \oplus u_{n}=f_{n} \oplus u_{n}$. Hence, Theorem 2.4 implies that $\left(\left\{f_{n} \oplus u_{n}: n \in\right.\right.$ I],$\left.T \lambda_{1} \oplus \lambda_{2}\right)$ is a Riesz baser on $H \oplus M$.

Lemma 2.6. Let $\left(\left\{u_{n}: n \in \mathbb{I}\right\}, \lambda_{2}\right)$ be a Riesz baser on $H$ and let $P$ be a projection from $H$ onto a subspace $P H$ of $H$, then $\left\{P u_{n}: n \in \mathbb{J}\right\}$ is a framer on $P H$.

Proof. Let $T: H \rightarrow H$ be a bounded invertible operator on $H$, and let $Q$ be a selfadjoint projection, set $P=T Q T^{-1}$ and let $v_{n}=T^{-1} u_{n}$, another Riesz baser on $H$. For $x \in Q H$, we have $\sum_{n}\left|\left\langle x, Q v_{n}\left(T^{-1} \lambda_{2}\right)\right\rangle\right|^{2}=\sum_{n}\left|\left\langle Q x, v_{n}\left(T^{-1} \lambda_{2}\right)\right\rangle\right|^{2}=\sum_{n}\left|\left\langle x, v_{n}\left(T^{-1} \lambda_{2}\right)\right\rangle\right|^{2}$, so by the definition of framer, $\left\{Q v_{n}: n \in \mathbb{J}\right\}$ is a framer on $H$. Now, since $T_{\mid Q H}$ is a bounded invertible operator from $Q H$ onto $P H$, so $\left\{T Q v_{n}: n \in \mathbb{D}\right\}$ is a framer on $P H$. 
THeOREm 2.7. A pair $\left(\left\{f_{n}: n \in \mathbb{I}\right\}, \lambda_{1}\right)$ is a framer on a Hilbert space $H$ if and only if there exist a Hilbert space $M$ and a normal framer $\left(\left\{u_{n}: n \in \mathbb{J}\right\}, \lambda_{2}\right)$ on $M$ such that $\left(\left\{f_{n} \oplus u_{n}\right.\right.$ : $\left.n \in \mathbb{D}\}, \lambda_{1} \oplus \lambda_{2}\right)$ is a Riesz baser on $H \oplus M$. In short, framers are precisely the inner direct summands of Riesz basers.

The proof is obvious.

Remark 2.8. The last theorem allows us to generalize the concept of framer in Banach spaces to be simply an inner direct summand of a Riesz baser. In this case, the index set should be $\mathbb{N}$. For instance, a Schauder framer would be an inner direct summand of a Schauder baser. Similarly, the bounded unconditional framer would be an inner direct summand of a bounded unconditional baser.

In the following theorems, we will prove that coisometries preserve the structure of framers, that is, the image of framers under coisometries are framers.

Theorem 2.9. Let $\left\{A_{j}\right\}_{j \in \mathbb{J}}$ be a family of coisometries on $H$ and let $\left(\left\{f_{j}: j \in \mathbb{J}\right\}, \lambda\right)$ be a framer for which $\left\{f_{j} \lambda\right\}_{j \in \mathbb{D}}$ is a frame with frame bounds $\left\{B_{1}, B_{2}\right\}$. Set $C_{j}=A_{j} f_{j}$, then $\left(\left\{C_{j}\right.\right.$ : $j \in \mathbb{J}\}, \lambda)$ is a framer with framer bounds $\left\{B_{1}, B_{2}\right\}$. If $\left(\left\{f_{j}: j \in \mathbb{J}\right\}, \lambda\right)$ is tight (normal), then $\left(\left\{C_{j}: j \in \mathbb{I}\right\}, \lambda\right)$ is tight (normal), respectively.

Proof. Let $x \in H$ be given, then $\sum_{j}\left|\left\langle x, C_{j} \lambda\right\rangle\right|^{2}=\sum_{j}\left|\left\langle x, A_{j} f_{j}(\lambda)\right\rangle\right|^{2}=\sum_{j}\left|\left\langle A_{j}^{*} x, f_{j} \lambda\right\rangle\right|^{2}$. Since $\left(\left\{f_{j}: j \in \mathbb{J}\right\}, \lambda\right)$ is a framer for which $\left\{f_{j} \lambda\right\}_{j \in \rrbracket}$ is a frame with frame bounds $\left\{B_{1}, B_{2}\right\}$, so $B_{1}\left\|A_{j}^{*} x\right\|^{2}<\sum_{j}\left|\left\langle A_{j}^{*} x, f_{j} \lambda\right\rangle\right|^{2}<B_{2}\left\|A_{j}^{*} x\right\|^{2}$. But $A_{j}$ is coisometry, for all $j$, hence $\left\|A_{j}^{*} x\right\|=\|x\|$, and then $B_{1}\|x\|^{2}<\sum_{j}\left|\left\langle A_{j}^{*} x, f_{j} \lambda\right\rangle\right|^{2}<B_{2}\|x\|^{2}$, or $B_{1}\|x\|^{2}<\sum_{j}\left|\left\langle x, C_{j} \lambda\right\rangle\right|^{2}<$ $B_{2}\|x\|^{2}$. So $\left(\left\{C_{j}: j \in \mathbb{J}\right\}, \lambda\right)$ is a framer with bounds $\left\{B_{1}, B_{2}\right\}$.

If $\left(\left\{f_{j}: j \in \mathbb{J}\right\}, \lambda\right)$ is a tight framer, then a similar calculation shows that $\sum_{j} \mid\langle x$, $\left.C_{j} \lambda\right\rangle\left.\right|^{2}=\sum_{j}\left|\left\langle A_{j}^{*} x, f_{j} \lambda\right\rangle\right|^{2}=B_{1}\left\|A_{j}^{*} x\right\|^{2}=B_{1}\|x\|^{2}$, that is, $\left(\left\{C_{j}: j \in \mathbb{I}\right\}, \lambda\right)$ is a tight framer on $H$.

And finally, if $\left(\left\{f_{j}: j \in \mathbb{\rrbracket}\right\}, \lambda\right)$ is normal, then $\left(\left\{C_{j}: j \in \mathbb{J}\right\}, \lambda\right)$ is a normal framer on $H$.

We can generalize this theorem as follows.

Theorem 2.10. Let $\left\{A_{j}^{1}\right\}_{j \in \rrbracket},\left\{A_{j}^{2}\right\}_{j \in \rrbracket}, \ldots,\left\{A_{j}^{n}\right\}_{j \in \mathbb{J}}$ be families of coisometries, and let $\left(\left\{f_{j}\right.\right.$ : $j \in \mathbb{D}\}, \lambda)$ be a framer with framer bounds $\left\{B_{1}, B_{2}\right\}$. If $C_{j}=A_{j}^{1} A_{j}^{2} \cdots A_{j}^{n} f_{j}$, then $\left(\left\{C_{j}: j \in\right.\right.$ $\mathbb{I}\}, \lambda)$ is a framer with framer bounds $\left\{B_{1}, B_{2}\right\}$. If $\left(\left\{f_{j}: j \in \mathbb{D}\right\}, \lambda\right)$ is tight (normal), then $\left(\left\{C_{j}: j \in \mathbb{D}\right\} \lambda\right)$ is tight (normal), respectively.

Proof. The proof is an easy consequence by Theorem 2.9 and induction.

Remark 2.11. For a tight framer $\left(\left\{f_{j}: j \in \mathbb{J}\right\}, \lambda\right)$ with framer bound $A$, the reconstruction formula is $x=(1 / A) \sum_{j}\left\langle x, f_{j} \lambda\right\rangle f_{j} \lambda$. If framer is not tight, then there is a similar reconstruction formula in terms of dual framers.

Let $\left(\left\{f_{j}: j \in \mathbb{D}\right\}, \lambda\right)$ be given. A framer $\left(\left\{g_{j}: j \in \mathbb{J}\right\}, \lambda\right)$ is called a dual framer of $\left(\left\{f_{j}\right.\right.$ : $j \in \mathbb{I}\}, \lambda)$ if for each $x \in H, x=\sum_{j}\left\langle x, g_{j} \lambda\right\rangle f_{j} \lambda$.

Theorem 2.12. Let $\left(\left\{f_{j}: j \in \mathbb{\rrbracket}\right\}, \lambda\right)$ be a framer on a Hilbert space $H$. Then there exists a unique operator $S: H \rightarrow H$ such that $x=\sum_{j \in N}\left\langle x, S f_{j} \lambda\right\rangle f_{j} \lambda$, for all $x \in H$. If $A: H \rightarrow K$ is any invertible operator for some Hilbert space $K$ such that $\left(\left\{A f_{j}: j \in \mathbb{D}\right\}, \lambda\right)$ is a normal framer, then $S=A^{*} A$. In particular, $S$ is an invertible positive operator. 
Proof. Since $\left(\left\{f_{j}: j \in \mathbb{J}\right\}, \lambda\right)$ is a framer, so $\left\{f_{j} \lambda: j \in \mathbb{J}\right\}$ is a frame for $H$, now the theorem is trivial.

We call $\left(\left\{S f_{j}: j \in \mathbb{J}\right\}, \lambda\right)$ the canonical dual of $\left(\left\{f_{j}: j \in \mathbb{\rrbracket}\right\}, \lambda\right)$, and for each $x \in H$, one can write $x=\sum_{j \in N}\left\langle x, S f_{j} \lambda\right\rangle f_{j} \lambda=\sum_{j \in N}\left\langle x, f_{j} \lambda\right\rangle S f_{j} \lambda$.

The last theorem proves that the canonical dual of each wavelet-type frame can be of wavelet-type as the following theorem proves the same results for the dual of a frame.

Theorem 2.13. Let $\left(\left\{f_{j}: j \in \mathbb{J}\right\}, h\right)$ and $\left(\left\{g_{j}: j \in \mathbb{J}\right\}, \lambda\right)$ be framers on a Hilbert space $H$, such that $x=\sum_{j}\left\langle x, g_{j} \lambda\right\rangle f_{j} h$ for all $x \in H$. Then $x=\sum_{j}\left\langle x, f_{j} h\right\rangle g_{j} \lambda$, for all $x \in H$.

The proof is obvious.

\section{Complement in framers and basers}

This section investigates some additional properties of framers and basers. First, we give some definitions.

Definition 3.1. Let $\left(\left\{f_{j}: j \in \mathbb{J}\right\}, \lambda_{1}\right)$ be a framer on a Hilbert space $H$. A framer $\left(\left\{g_{j}: j \in\right.\right.$ $\left.\sqrt{ }\}, \lambda_{2}\right)$ on a Hilbert space $M$ is called a complement to $\left(\left\{f_{j}: j \in \mathbb{J}\right\}, \lambda_{1}\right)$ if $\left(\left\{f_{j} \oplus g_{j}: j \in\right.\right.$ ป\},$\left.\lambda_{1} \oplus \lambda_{2}\right)$ is a Riesz baser on $H \oplus M$. Note that by Theorem 2.7, the complement to each framer $\left(\left\{f_{j}: j \in \mathbb{D}\right\}, \lambda_{1}\right)$ exists.

If $\left(\left\{f_{j}: j \in \mathbb{I}\right\}, \lambda_{1}\right)$ is a normal framer, then a normal framer $\left(\left\{g_{j}: j \in \mathbb{D}\right\}, \lambda_{2}\right)$ on a Hilbert space $M$ is called a strong complement to $\left(\left\{f_{j}: j \in \mathbb{J}\right\}, \lambda_{1}\right)$ if $\left(\left\{f_{j} \oplus g_{j}: j \in \mathbb{J}\right\}, \lambda_{1} \oplus\right.$ $\left.\lambda_{2}\right)$ is an orthonormal baser on $H \oplus M$.

Two framers $\left(\left\{f_{j}: j \in \mathbb{J}\right\}, \lambda_{1}\right)$ and $\left(\left\{g_{j}: j \in \mathbb{J}\right\}, \lambda_{2}\right)$ on Hilbert spaces $H, K$, respectively, are similar if there exists a linear bounded invertible operator $T: H \rightarrow K$ such that $T f_{j} T^{*}=g_{j}$ for each $j \in J$. In this case, if $T$ is unitary, then $\left(\left\{f_{j}: j \in \mathbb{I}\right\}, \lambda_{1}\right)$ and $\left(\left\{g_{j}: j \in \mathbb{I}\right\}, \lambda_{2}\right)$ are called unitarily equivalent.

A framer $\left(\left\{g_{j}: j \in \mathbb{I}\right\}, \lambda_{2}\right)$ is called strong complement to $\left(\left\{f_{j}: j \in \mathbb{D}\right\}, \lambda_{1}\right)$ if there exist a pair of strong complement normal framers $\left(\left\{f_{j}^{\prime}: j \in \mathbb{I}\right\}, \lambda_{1}\right)$ and $\left(\left\{g_{j}^{\prime}: j \in \mathbb{I}\right\}, \lambda_{2}\right)$ and linear bounded invertible operators $T_{1}$ and $T_{2}$ such that $f_{j}^{\prime}=T_{1} f_{j} T_{1}^{*}$ and $g_{j}^{\prime}=T_{2} g_{j} T_{2}^{*}$ for all $j \in \mathbb{J}$.

Theorem 3.2. Let $\left(\left\{f_{j}: j \in \mathbb{I}\right\}, \lambda_{1}\right)$ be a framer on $H$, and let $\left(\left\{g_{j}: j \in \mathbb{I}\right\}, \lambda_{2}\right)$ and $\left(\left\{e_{j}:\right.\right.$ $\left.j \in \mathbb{J}\}, \lambda_{3}\right)$ be strong complements to $\left(\left\{f_{j}: j \in \mathbb{J}\right\}, \lambda_{1}\right)$ in Hilbert spaces $M, N$, respectively. Then there exists an invertible operator $\Delta: M \rightarrow N$ such that $e_{j}=\Delta g_{j} \Delta^{*}$ for all $j \in \mathbb{J}$.

The proof is immediate.

In the next theorem, we will show that the strong complementary of normal framers are preserved under coisometries.

Theorem 3.3. Let $\left(\left\{f_{j}: j \in \mathbb{I}\right\}, \lambda_{1}\right)$ be a normal framer on $H$, and let $\left\{A_{j}: j \in \mathbb{I}\right\}$ be a family of coisometries operators on $H$. Then there exist a Hilbert space $M$ with normal framer $\left(\left\{g_{j}: j \in \mathbb{I}\right\}, \lambda_{2}\right)$, and a family of coisometries operators $\left\{B_{j}: j \in \mathbb{I}\right\}$ on $M$ such that $\left(\left\{g_{j}\right.\right.$ : $\left.j \in \mathbb{J}\}, \lambda_{2}\right)$ is a strong complement to $\left(\left\{f_{j}: j \in \mathbb{J}\right\}, \lambda_{1}\right)$ and $\left(\left\{B_{j} g_{j}: j \in \mathbb{I}\right\}, \lambda_{2}\right)$ is a strong complement to $\left(\left\{A_{j} f_{j}: j \in \mathbb{J}\right\}, \lambda_{1}\right)$.

Proof. Let $\left(\left\{f_{j}: j \in \mathbb{J}\right\}, \lambda_{1}\right)$ be a normal framer and $\left\{A_{j}: j \in \mathbb{J}\right\}$ is a family of coisometries. With notations as in Theorem 2.2, since $\theta: H \rightarrow \theta_{0}(H)$ is unitary, so $\theta \oplus I$ and $\theta^{-1} \oplus I$ 
are unitaries. Hence by Theorems 2.3 and 2.9, there exist a Hilbert space $M$ and a normal framer $\left(\left\{e_{j}: j \in \mathbb{J}\right\}, \lambda_{3}\right)$ such that $\left(\left\{A_{j} f_{j} \oplus e_{j}: j \in \mathbb{J}\right\}, \lambda_{1}+\lambda_{3}\right)$ is an orthonormal baser on $H \oplus M$. On the other hand, since $\left(\left\{f_{j}: j \in \mathbb{I}\right\}, \lambda_{1}\right)$ is normal framer, so there exists a normal framer $\left(\left\{g_{j}: j \in \mathbb{J}\right\}, \lambda_{2}\right)$ which is a strong complement to $\left(\left\{f_{j}: j \in \mathbb{J}\right\}, \lambda_{1}\right)$, that is, $\left(\left\{f_{j} \oplus g_{j}: j \in \mathbb{J}\right\}, \lambda_{1} \oplus \lambda_{2}\right)$ is an orthonormal baser on $H \oplus M$. Set $B_{j}=e_{j} g_{j}^{-1}$ since $\left\|B_{j}^{*} y\right\|^{2}=\sum_{j \in \mathbb{J}}\left|\left\langle B_{j}^{*} y, g_{j} \lambda_{3}\right\rangle\right|^{2}=\sum_{j \in \mathbb{J}}\left|\left\langle y, B_{j} g_{j} \lambda_{3}\right\rangle\right|^{2}=\sum_{j \in \mathbb{l}}\left|\left\langle y, e_{j} \lambda_{3}\right\rangle\right|^{2}=\|y\|^{2}$. So $B_{j}$, for each $j \in \mathbb{J}$, is a bounded invertible coisometry. Now, $B_{j} g_{j}=e_{j}$ and $\left(\left\{e_{j}: j \in \mathbb{J}\right\}, \lambda_{3}\right)$ is a strong complement to $\left(\left\{A_{j} f_{j}: j \in \mathbb{J}\right\}, \lambda_{1}\right)$, so $\left(\left\{B_{j} g_{j}: j \in \mathbb{J}\right\}, \lambda_{3}\right)$ is a strong complement to $\left(\left\{A_{j} f_{j}: j \in \mathbb{D}\right\}, \lambda_{1}\right)$.

A similar conclusion holds for framers as follows.

Theorem 3.4. Let $\left(\left\{f_{j}: j \in \mathbb{J}\right\}, \lambda_{1}\right)$ be a framer on $H$, and let $\left\{A_{j}: j \in \mathbb{J}\right\}$ be a family of coisometry operators on $H$. Then there exist a Hilbert space $M$ with normal framer $\left(\left\{g_{j}: j \in\right.\right.$ $\left.\mathbb{I}\}, \lambda_{2}\right)$, and a family of coisometry operators $\left\{C_{j}: j \in \mathbb{J}\right\}$ on $M$ such that $\left(\left\{g_{j}: j \in \mathbb{J}\right\}, \lambda_{2}\right)$ is complement to $\left(\left\{f_{j}: j \in \mathbb{J}\right\}, \lambda_{1}\right)$ and $\left(\left\{C_{j} g_{j}: j \in \mathbb{I}\right\}, \lambda_{3}\right)$ is complement to $\left(\left\{A_{j} f_{j}: j \in\right.\right.$ ป $\left.\}, \lambda_{1}\right)$.

Proof. Let $\left(\left\{f_{j}: j \in \mathbb{J}\right\}, \lambda_{1}\right)$ be a framer and $\left\{A_{j}: j \in \mathbb{J}\right\}$ are coisometries, so $\left(\left\{A_{j} f_{j}: j \in\right.\right.$ $\left.\mathbb{I}\}, \lambda_{1}\right)$ is a framer on $H$. On the other hand, there are normal framers $\left(\left\{g_{j}: j \in \mathbb{I}\right\}, \lambda_{2}\right)$ and $\left(\left\{e_{j}: j \in \mathbb{J}\right\}, \lambda_{3}\right)$ on $M$ such that $\left(\left\{f_{j} \oplus g_{j}: j \in \mathbb{J}\right\}, \lambda_{1} \oplus \lambda_{2}\right)$ and $\left(\left\{A_{j} f_{j} \oplus e_{j}: j \in \mathbb{J}\right\}, \lambda_{1} \oplus\right.$ $\left.\lambda_{3}\right)$ are Riesz basers on $H \oplus M$, that is, $\left(\left\{g_{j}: j \in \mathbb{I}\right\}, \lambda_{2}\right)$ is complement to $\left(\left\{f_{j}: j \in \mathbb{I}\right\}, \lambda_{1}\right)$.

For the other part of theorem, set $C_{j}=e_{j} g_{j}^{-1}$, then a calculation such as Theorem 3.3 shows that $\left\{C_{j}: j \in \mathbb{I}\right\}$ are coisometries and since $\left(\left\{A_{j} f_{j} \oplus C_{j} g_{j}: j \in \mathbb{J}\right\}, \lambda_{1} \oplus \lambda_{3}\right)$ is a Riesz baser on $H \oplus M$, hence $\left(\left\{C_{j} g_{j}: j \in \mathbb{I}\right\}, \lambda_{3}\right)$ is a complement to $\left(\left\{A_{j} f_{j}: j \in \mathbb{J}\right\}, \lambda_{1}\right)$.

In the following, we will prove that the strong complement of a wavelet-type normalized tight frame is of wavelet-type.

Theorem 3.5. Let $\left\{x_{j}: j \in \mathbb{J}\right\}$ be a normalized tight wavelet-type frame for a Hilbert space $H$, and let $\left\{y_{j}: j \in \mathbb{J}\right\}$ be a strong complement to $\left\{x_{j}: j \in \mathbb{J}\right\}$ in the frame sense, then $\left\{y_{j}:\right.$ $j \in \mathbb{D}\}$ is a wavelet-type frame.

Proof. Let $\left\{x_{j}: j \in \mathbb{J}\right\}$ be a normalized tight wavelet-type frame for a Hilbert space $H$, so there exists a normal framer $\left(\left\{f_{j}: j \in \mathbb{J}\right\}, \lambda_{1}\right)$ on $H$ such that $x_{j}=f_{j} \lambda_{1}$ for all $j \in \mathbb{J}$. Since $\left(\left\{f_{j}: j \in \mathbb{I}\right\}, \lambda_{1}\right)$ is normal framer, so there exists a normal framer $\left(\left\{g_{j}: j \in \mathbb{I}\right\}, \lambda_{2}\right)$ such that $\left(\left\{f_{j} \oplus g_{j}: j \in \mathbb{D}\right\}, \lambda_{1} \oplus \lambda_{2}\right)$ is an orthonormal baser on $H \oplus M$. So $\left\{g_{j} \lambda_{2}: j \in \mathbb{I}\right\}$ is a strong complement to $\left\{f_{j} \lambda_{1}: j \in \mathbb{I}\right\}$ in the sense of frames. But $\left\{y_{j}: j \in \mathbb{J}\right\}$ is another strong complement to $\left\{f_{j} \lambda_{1}: j \in \mathbb{J}\right\}$, so by Theorem 3.2, there exists a unitary operator $U: M \rightarrow N$ such that $y_{j}=U g_{j}\left(\lambda_{2}\right)$ and $y_{j}=U g_{j} U^{*}\left(\lambda_{3}\right)$, for $\lambda_{3} \in N$. Set $e_{j}=U g_{j} U^{*}$, then $\left(\left\{e_{j}: j \in \mathbb{I}\right\}, \lambda_{3}\right)$ is a normal framer and $e_{j} \lambda_{3}=y_{j}$, that is, $\left\{y_{j}: j \in \mathbb{D}\right\}$ is wavelet-type.

For the case that $\left\{x_{j}: j \in \mathbb{I}\right\}$ is a general wavelet-type frame, first we prove the following lemma, stated that the similar of a wavelet-type frame is of wavelet-type.

Lemma 3.6. Let $\left\{x_{j}: j \in \mathbb{I}\right\}$ be a wavelet-type frame for a Hilbert space $H$, and let $\left\{y_{j}: j \in\right.$ I\} be a frame similar to it. Then $\left\{y_{j}: j \in \mathbb{J}\right\}$ is wavelet-type. 
Proof. Since $\left\{x_{j}: j \in \mathbb{J}\right\}$ is similar to $\left\{y_{j}: j \in \mathbb{J}\right\}$, there exists a bounded invertible operator $T: H \rightarrow H$ such that $T x_{j}=y_{j}$, since $\left\{x_{j}: j \in \mathbb{J}\right\}$ is a wavelet-type frame, there exists a framer $\left(\left\{f_{j}: j \in \mathbb{I}\right\}, \lambda_{1}\right)$ such that $x_{j}=f_{j} \lambda_{1}$, hence $T f_{j} \lambda_{1}=y_{j}$. Now set $g_{j}=T f_{j}$, then $\left(\left\{g_{j}: j \in \mathbb{I}\right\}, \lambda_{1}\right)$ is a framer and $g_{j} \lambda_{1}=y_{j}$, for all $j \in \mathbb{J}$. Thus, $\left\{y_{j}: j \in \mathbb{I}\right\}$ is wavelettype.

The following theorem shows that the strong complement of each wavelet-type frame is of wavelet-type.

Theorem 3.7. Let $\left\{x_{j}: j \in \mathbb{J}\right\}$ be a wavelet-type frame for a Hilbert space $H$, let $\left\{y_{j}: j \in \mathbb{D}\right\}$ be a strong complement to $\left\{x_{j}: j \in \mathbb{D}\right\}$, then $\left\{y_{j}: j \in \mathbb{D}\right\}$ is wavelet-type.

Proof. Since $\left\{x_{j}: j \in \mathbb{\rrbracket}\right\}$ and $\left\{y_{j}: j \in \mathbb{J}\right\}$ are strong complements, so by the definition, there exists normalized tight frames $\left\{x_{j}^{\prime}: j \in \mathbb{D}\right\}$ and $\left\{y_{j}^{\prime}: j \in \mathbb{J}\right\}$ such that $\left\{x_{j}: j \in \mathbb{D}\right\}$ is similar to $\left\{x_{j}^{\prime}: j \in \mathbb{J}\right\},\left\{y_{j}: j \in \mathbb{J}\right\}$ is similar to $\left\{y_{j}^{\prime}: j \in \mathbb{J}\right\}$, and $\left\{x_{j}^{\prime} \oplus y_{j}^{\prime}: j \in \mathbb{J}\right\}$ is an orthonormal basis. Since $\left\{x_{j}: j \in \mathbb{J}\right\}$ is a wavelet-type frame and $\left\{x_{j}^{\prime}: j \in \mathbb{l}\right\}$ is similar to it, so by Lemma 3.6, the frame $\left\{x_{j}^{\prime}: j \in \mathbb{J}\right\}$ is wavelet-type. On the other hand, since $\left\{y_{j}^{\prime}: j \in \mathbb{D}\right\}$ is a strong complement to $\left\{x_{j}^{\prime}: j \in \mathbb{J}\right\}$ and $\left\{x_{j}^{\prime}: j \in \mathbb{J}\right\}$ is wavelet-type, so by Theorem 3.5, the frame $\left\{y_{j}^{\prime}: j \in \mathbb{J}\right\}$ is wavelet-type. Therefore, there exists a normal framer $\left(\left\{g_{j}: j \in \mathbb{I}\right\}, \lambda_{2}\right)$ such that $g_{j} \lambda_{2}=y_{j}^{\prime}$. Since $\left\{y_{j}^{\prime}: j \in \mathbb{I}\right\}$ and $\left\{y_{j}: j \in \mathbb{I}\right\}$ are similar, so there is a bounded invertible operator $T$ such that $T y_{j}=y_{j}^{\prime}=g_{j} \lambda_{2}$. Thus $y_{j}=T^{-1} g_{j} \lambda_{2}$. Now set $e_{j}=T^{-1} g_{j}$, then $y_{j}=e_{j} \lambda_{2}$. That is, $\left\{y_{j}: j \in \mathbb{J}\right\}$ is wavelet-type.

Definition 3.8. Let $\left(\left\{f_{j}: j \in \mathbb{I}\right\}, \lambda_{1}\right)$ and $\left(\left\{g_{j}: j \in \mathbb{I}\right\}, \lambda_{2}\right)$ be normal framers on Hilbert spaces $H$ and $K$, respectively. $\left(\left\{f_{j}: j \in \mathbb{J}\right\}, \lambda_{1}\right)$ and $\left(\left\{g_{j}: j \in \mathbb{J}\right\}, \lambda_{2}\right)$ are strongly disjoint (strongly completely disjoint) if $\left(\left\{f_{j} \oplus g_{j}: j \in \mathbb{I}\right\}, \lambda_{1} \oplus \lambda_{2}\right)$ is a normal framer (orthonormal baser) on $H \oplus M$.

If $\left(\left\{f_{j}: j \in \mathbb{D}\right\}, \lambda_{1}\right)$ and $\left(\left\{g_{j}: j \in \mathbb{J}\right\}, \lambda_{2}\right)$ are framers (not necessarily tight or normal), $\left(\left\{f_{j}: j \in \mathbb{J}\right\}, \lambda_{1}\right)$ and $\left(\left\{g_{j}: j \in \mathbb{J}\right\}, \lambda_{2}\right)$ are disjoint if $\left(\left\{f_{j} \oplus g_{j}: j \in \mathbb{J}\right\}, \lambda_{1} \oplus \lambda_{2}\right)$ is a framer on $H \oplus M$.

Two framers $\left(\left\{f_{j}: j \in \mathbb{I}\right\}, \lambda_{1}\right)$ and $\left(\left\{g_{j}: j \in \mathbb{J}\right\}, \lambda_{2}\right)$ are called strongly disjoint (strongly completely disjoint) if they are similar to a pair of strongly disjoint (strongly completely disjoint) normal framers.

Finally our last task in this paper is to prove that the strongly disjointness of each wavelet-type normalized tight frame is of wavelet-type. This will be shown in the following theorem.

Theorem 3.9. Let $\left\{x_{j}: j \in \mathbb{J}\right\}$ be a wavelet-type normalized tight frame for a Hilbert space $H$, and let $\left\{y_{j}: j \in \mathbb{J}\right\}$ be a strongly disjoint frame to it. Then there exists a normalized tight frame $\left\{z_{j}: j \in \mathbb{J}\right\}$ such that $\left\{y_{j} \oplus z_{j}: j \in \mathbb{I}\right\}$ is of wavelet-type.

Proof. Let $\left\{x_{j}: j \in \mathbb{J}\right\}$ be a wavelet-type normalized tight frame. So there exists a normal framer $\left(\left\{f_{j}: j \in \mathbb{I}\right\}, \lambda_{1}\right)$ such that $x_{j}=f_{j} \lambda_{1}$ for all $j \in \mathbb{J}$. Since $\left\{y_{j}: j \in \mathbb{J}\right\}$ is strongly disjoint to $\left\{x_{j}: j \in \mathbb{I}\right\}$, thus $\left\{x_{j} \oplus y_{j}: j \in \mathbb{I}\right\}$ is a normalized tight frame and there exists a normalized tight frame $\left\{z_{j}: j \in \mathbb{J}\right\}$ such that $\left\{\left(x_{j} \oplus y_{j}\right) \oplus z_{j}: j \in \mathbb{J}\right\}$ is an orthonormal basis. 
But $\left\{\left(x_{j} \oplus y_{j}\right) \oplus z_{j}: j \in \mathbb{D}\right\}$ and $\left\{x_{j} \oplus\left(y_{j} \oplus z_{j}\right): j \in \mathbb{J}\right\}$ are unitarily equivalent, hence $\left\{x_{j} \oplus\left(y_{j} \oplus z_{j}\right): j \in \mathbb{\rrbracket}\right\}$ is an orthonormal basis. Since $\left\{x_{j}: j \in \mathbb{J}\right\}$ is a normalized tight frame, so $\left\{y_{j} \oplus z_{j}: j \in \mathbb{J}\right\}$ is a normalized tight frame, which is a strong complement to $\left\{x_{j}: j \in \mathbb{D}\right\}$. Now $\left\{x_{j}: j \in \mathbb{D}\right\}$ is a wavelet-type normalized tight frame, so by Theorem 3.7, the frame $\left\{y_{j} \oplus z_{j}: j \in \mathbb{J}\right\}$ is wavelet-type as required.

\section{References}

[1] J. J. Benedetto and D. F. Walnut, Gabor frames for $L^{2}$ and related spaces, Wavelets: Mathematics and Applications, Stud. Adv. Math., CRC Press, Florida, 1994, pp. 97-162.

[2] Y. M. Berezansky, Z. G. Sheftel, and G. F. Us, Functional Analysis. Vol. I, Operator Theory: Advances and Applications, vol. 85, Birkhäuser, Basel, 1996, translated from the 1990 Russian original by Peter V. Malyshev.

[3] _ Functional Analysis. Vol. II, Operator Theory: Advances and Applications, vol. 86, Birkhäuser, Basel, 1996, translated from the 1990 Russian original by Peter V. Malyshev.

[4] X. Dai and D. R. Larson, Wandering vectors for unitary systems and orthogonal wavelets, Mem. Amer. Math. Soc. 134 (1998), no. 640, viii+68.

[5] I. Daubechies, Ten Lectures on Wavelets, CBMS-NSF Regional Conference Series in Applied Mathematics, vol. 61, SIAM, Pennsylvania, 1992.

[6] L. Debnath, Wavelet Transforms and Their Applications, Birkhäuser, Massachusetts, 2002.

[7] G. B. Folland, A Course in Abstract Harmonic Analysis, Studies in Advanced Mathematics, CRC Press, Florida, 1995.

[8] D. Han and D. R. Larson, Frames, bases and group representations, Mem. Amer. Math. Soc. 147 (2000), no. 697, $\mathrm{x}+94$.

[9] J. R. Holub, Pre-frame operators, Besselian frames, and near-Riesz bases in Hilbert spaces, Proc. Amer. Math. Soc. 122 (1994), no. 3, 779-785.

[10] R. V. Kadison and J. R. Ringrose, Fundamentals of the Theory of Operator Algebras. Vol. I: Elementary Theory, Pure and Applied Mathematics, vol. 100, Academic Press, New York, 1983.

M. M. Shamooshaky: Department of Mathematics, Imam Hossein University, P.O. Box 16895-198, Tehran, Iran

E-mail address: mshmshki@ihu.ac.ir 


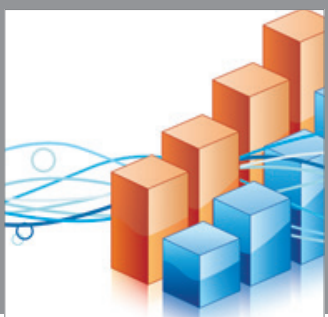

Advances in

Operations Research

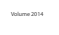

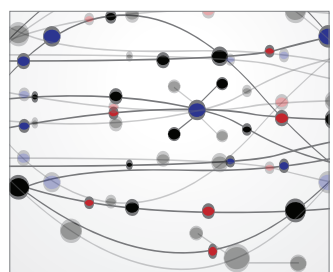

\section{The Scientific} World Journal
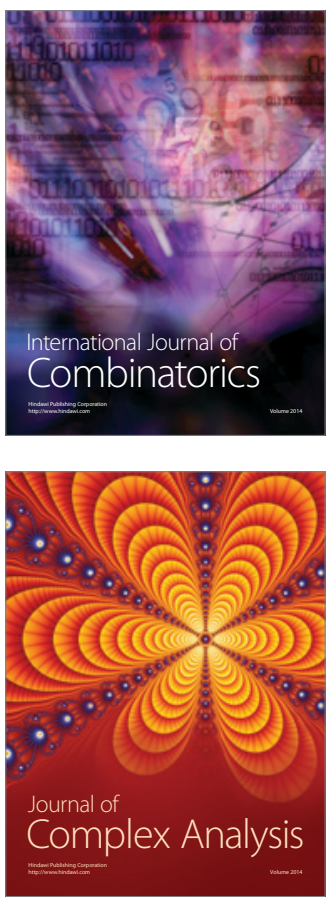

International Journal of

Mathematics and

Mathematical

Sciences
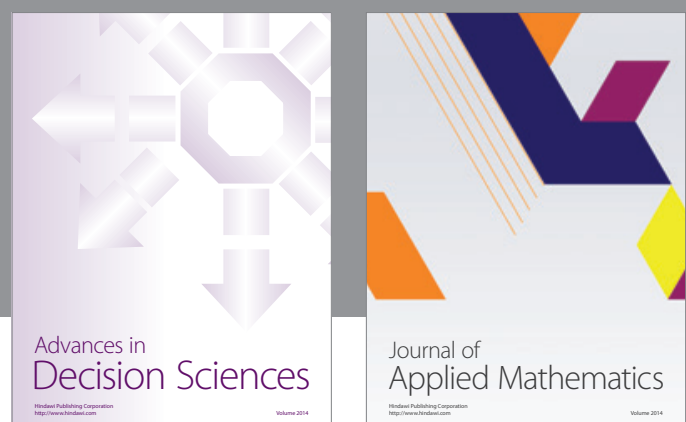

Journal of

Applied Mathematics
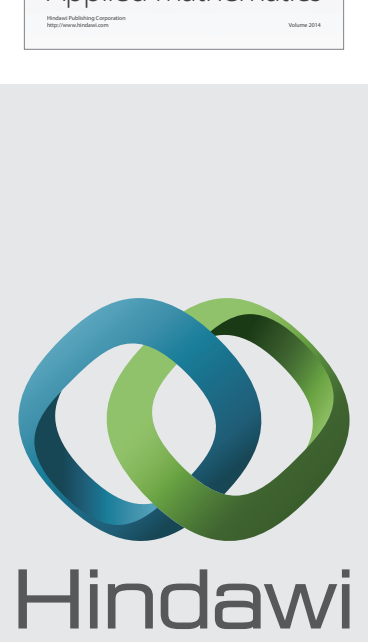

Submit your manuscripts at http://www.hindawi.com
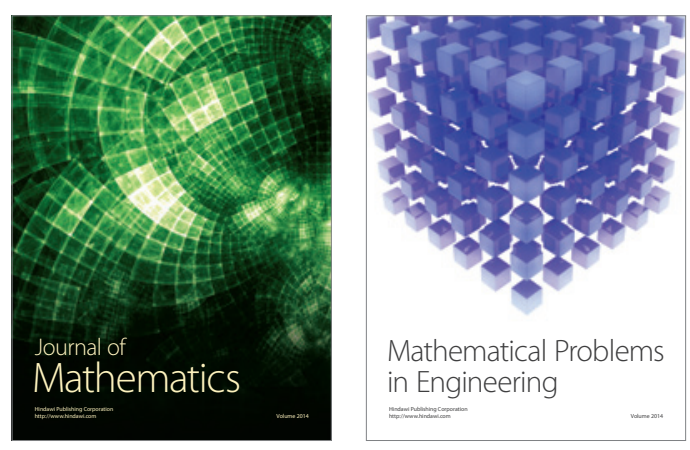

Mathematical Problems in Engineering
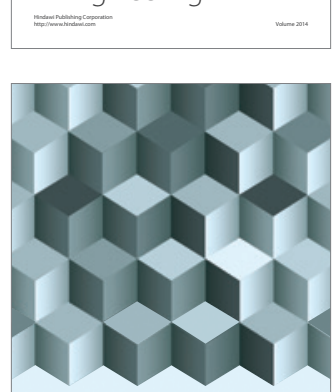

Journal of

Function Spaces
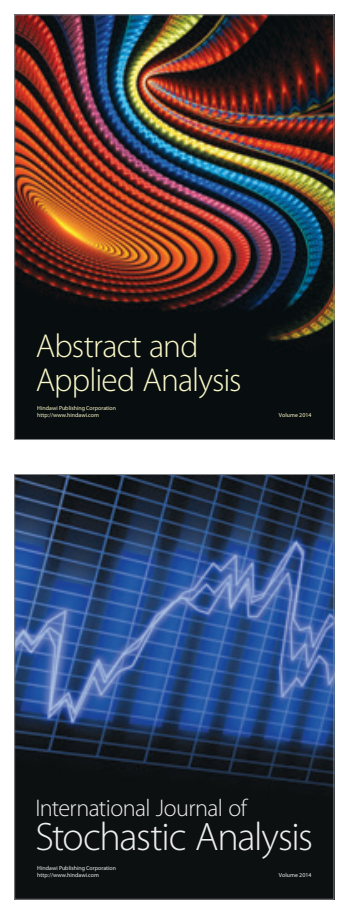

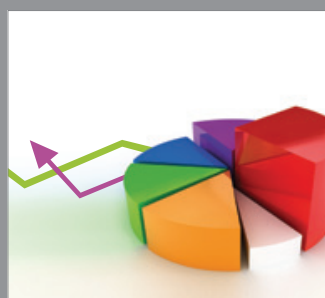

ournal of

Probability and Statistics

Promensencen
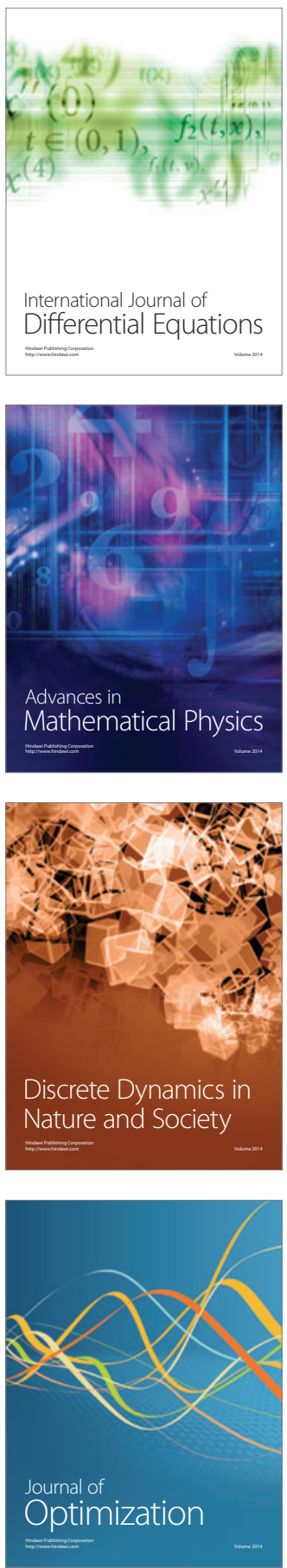